\title{
Le carrefour thérapeutique : médecine, techniques et pouvoirs dans l'Europe moderne
}

The "Therapeutic Crossroads": Medicine, Technology and Power in Early Modern Europe

\section{Christelle Rabier}

\section{OpenEdition}

\section{Journals}

Édition électronique

URL : http://journals.openedition.org/artefact/342

DOI : $10.4000 /$ artefact.342

ISSN : 2606-9245

\section{Éditeur :}

Association Artefact. Techniques histoire et sciences humaines, Presses universitaires du Midi

\section{Édition imprimée}

Date de publication : 1 octobre 2016

Pagination : 83-95

ISBN : 978-2-7535-5174-9

ISSN : 2273-0753

\section{Référence électronique}

Christelle Rabier, «Le carrefour thérapeutique : médecine, techniques et pouvoirs dans l'Europe moderne », Artefact [En ligne], 4 | 2016, mis en ligne le 07 juillet 2017, consulté le 19 avril 2019. URL : http://journals.openedition.org/artefact/342 ; DOI : 10.4000/artefact.342 


\section{Le carrefour thérapeutique : médecine, techniques et pouvoirs dans l'Europe moderne}

Christelle RABIER*

\section{Résumé}

Les travaux récents en histoire de la médecine construisent un "carrefour thérapeutique » qui renouvelle l'histoire des techniques de l'Europe moderne. S'y croisent des perspectives nouvelles sur la commercialisation et sur l'appropriation de nouveaux ingrédients et de savoir-faire nouveaux dans la materia medica européenne, qui en retour transforment les théories médicales. Le point de vue technique conduit à réexaminer la périodisation, les lieux et les acteurs de la médicalisation.

Mots-clés : commerce, Europe moderne, histoire de la médecine, patient, techniques.

\section{Abstract. The " therapeutic crossroads ": medicine, technology and power in early modern Europe}

Recent literature in medical history has set up a " therapeutic crossroads » which set new perspectives on the history of early-modern European technology, that is commercialization of services as well as appropriation of new ingredients in European materia medica on artisanal know-how, which conversely transformed medical theory. The technological point of view offers new perspectives on the chronology, places and actors of medicalization.

Keywords : commerce, early-modern Europe, medical history, patient, techniques.

\footnotetext{
*. Christelle Rabier, maîtresse de conférences à l'École des hautes études en sciences sociales [http:/ / esopp. ehess.fr/index.php?716], a développé des recherches sur l'histoire technique et économique de la médicalisation européenne à l'époque moderne. Elle a notamment édité Fitting for health. The economy of medical technologies in early moderne Europe, numéro spécial de Technology and Culture, vol. 53, $\mathrm{n}^{\circ} 3$ (2013). Contact [christelle. rabier@gmail.com]. Mes plus vifs remerciements vont à l'équipe d'Artefact, à Samir Boumediene et à Thomas Le Roux pour leur patiente générosité.
} 
L'histoire de la médecine moderne européenne, aussi étrange que cela puisse paraître et à la différence des études contemporaines, s'est longtemps écrite à l'écart de l'histoire des techniques ${ }^{1}$. Pour être plus juste, elle a développé une conception particulière de techniques dites « de gouvernement » par la médecine, regroupant sous ce terme un ensemble de dispositifs visant au contrôle politique des corps et des sujets. Cette perspective, dans la lignée des travaux de Michel Foucault, ne portait guère d'attention à la matérialité et aux gestes qui ont façonné la médicalisation des sociétés européennes.

Les historiens (et historiennes) de l'époque moderne, prenant des chemins de traverse, offrent désormais une réflexion originale sur les techniques de soin. Le "carrefour thérapeutique " résulte de plusieurs facteurs institution- nels et épistémologiques : l'incorporation de la médecine dans des études dites " de sciences et de techniques " (STS); le dialogue, renoué au cours des années 1990 entre conservateurs de musée et chercheurs en sciences et techniques, pour donner sens aux collections médicales; le souci, enfin, d'examiner l'activité des praticiens de la médecine au plus près des pratiques, en Europe et hors de ses frontières. Le propos qui suit entend analyser le point nodal qu'est l'activité thérapeutique pour comprendre les rapports entre médecine, sociétés et pouvoirs, à la charnière d'études portant sur la commercialisation et la matérialité des savoirs et savoir-faire thérapeutiques. Il invite, en retour, à reconsidérer les dynamiques de la médicalisation européenne.

\section{La " grande chaîne de l'achat ": techniques médicales et commercialisation}

Le « carrefour de la consommation » (Ruth Schwartz Cowan) représente un des tout premiers espaces de conceptualisation des rapports entre techniques, médecine et sociétés ${ }^{2}$. Constatant que les journaux londoniens au $\mathrm{XVIII}^{\mathrm{e}}$ siècle regorgent de publicités pour des remèdes de toute sorte, Roy Porter s'interroge sur le rôle des patients, à la fois destinataires et prescripteurs de services ${ }^{3}$ : il fallait, selon lui, replacer la médecine dans la réflexion sur l'accélération commerciale de l'époque moderne. L'attention aux chaînons de l'activité commerciale, pour plagier l'heureuse expression de Colin Jones sur la « grande chaîne de l'achat » (great chain of buying), a conduit à jeter un œil neuf sur les vendeurs de médecine et les techniques de commercialisation d'un certain nombre de produits. Avant même d'étudier les marchandises proprement dites, l'attention s'est d'abord portée sur ces " entrepreneurs médicaux » (Roy Porter), en particulier pour l'Italie. Là, les vendeurs ambulants $\mathrm{y}$ sont souvent soumis à autorisation et passibles de sanctions judiciaires ou pécuniaires pour pratique "illégale » de la médecine, ce qui permet de connaître avec une relative précision leurs activités et leurs modes opératoires, comme les remèdes mis en vente, en recensant leurs 
multiples fonctions - douleurs dentaires, maux multiples, blessures. Le « charlatanisme médical » (D. Gentilcore) définit un monde artisanal qui façonne des formes galéniques - onguents, huiles, etc. - et les transforme : essor de la distillation au $\mathrm{xVI}^{\mathrm{e}}$ siècle, développement des remèdes composés, différenciation des modes d'application ${ }^{4}$. Il s'agit aussi d'un monde commercial, dont les techniques de vente mises en œuvre sont dignes du marketing contemporain - rôle de la mise à prix, des emballages, des gammes de produits pour toucher une clientèle socialement diversifiée, prétentions à la nouveauté, création de marque, sans parler des publicités - et qui fait un usage assez remarquable des routes de commerce, des marchés et des foires pour la vente ambulante. L'innovation thérapeutique va ainsi de pair avec une transformation des techniques de communication et de vente.

Enseignes de boutique, placards, trade cards (cartes commerciales), encarts publicitaires, les nouvelles techniques $\mathrm{d}$ 'information façonnent les rencontres entre clients de médecine et fournisseurs de services à l'époque moderne ${ }^{5}$. Les travaux récents soulignent à quel point le médium imprimé a activement contribué à la marchandisation médicale et à la configuration des produits. Dans l'Angleterre géorgienne, la plupart des entreprises de presse ont des parts dans la revente de remèdes brevetés (proprietary medicines) dont ils font la publicité L'information sur les nouvelles techniques médicales prend de nouvelles formes qui incluent, outre les annonces dans la presse, les traités spécialisés qui se présentent sous la forme de manuel d'utilisation et les cours publics et privés, de plus en plus courus dans les capitales européennes. Entre les XVII ${ }^{\mathrm{e}}$ et $\mathrm{xVIII}^{\mathrm{e}}$ siècles, les traités eux-mêmes ne portent pas la marque d'une distinction entre science médicale et médecine commerciale; leurs pages abritent des catalogues d'ouvrages médicaux, de remèdes ou d'instruments; certains, destinés à un large public, sont des produits éditoriaux, aux auteurs mal déterminés, à tel point qu'on peut considérer les imprimés médicaux comme des marchandises thérapeutiques ${ }^{7}$. L'éducation médicale s'avère ainsi indissociable de l'essor de la commercialisation.

Le développement des réseaux de postes transforme la spatialisation de la relation thérapeutique, tant pour les consultations par lettre, l'acheminement des remèdes que pour la vente d'appareils 8 . Prenons un exemple récemment étudié à propos d'appareils orthopédiques pour contenir les hernies, dits «bandages herniaires ", dont l'essor de la commercialisation commence à la fin du xvir ${ }^{\mathrm{e}}$ siècle9. Au milieu du siècle suivant, depuis leur boutique d'arrière-cour du faubourg Saint-Honoré parisien où ils reçoivent clients et courrier, William Blakey et son épouse Elizabeth Aumerle développent une politique de vente particulièrement ambitieuse. Blakey affiche des placards dans sa rue, se déplace à la demande chez des particuliers, fait paraître des avis de nouveautés dans la presse en France et à Londres; il envoie des brochures publicitaires à d'anciens clients dans tout le royaume; il utilise le service d'agents commerciaux ou de praticiens médicaux - comme les chirurgiens hospitaliers - pour la revente et l'obtention de marchés dans l'armée ou les hôpitaux; il ouvre enfin une succur- 
sale à Londres. Blakey et Aumerle entretiennent une active correspondance avec leurs patients, originaires de France, mais aussi de Gand, Liège et Vienne. La créativité de ces dispositifs commerciaux qui concernent d'autres marchandises comme les remèdes, contribue à organiser des marchés segmentés mais coordonnés entre zones géographiques et classes sociales, ajoutant quelques maillons à la " grande chaîne de $\mathrm{l}^{\prime}$ achat ${ }^{10}$ ».

Dans ce marché médical moderne, les praticiens médicaux, par les relations de service qu'ils construisent, s'avèrent des pivots essentiels dans un monde d'échange européen. La correspondance des médecins s'avère être une source précieuse pour comprendre comment les praticiens, qui accompagnent leurs lettres d'échantillons de plantes, s'échangent de l'information sur les propriétés des plantes, par exemple, et la fabrication des remèdes, mais aussi les moyens de s'en procurer; prescripteurs, ils représentent un maillon entre fabricants et clients, exerçant à la fois le rôle de conseil auprès de particuliers, agents commerciaux d'autres médecins, acheteurs pour le compte des hôpitaux ou de maisons aristocratiques, etc. ${ }^{11}$. Espaces traditionnels de revente dans les villes européennes, les apothicaireries représentent désormais des lieux d'innovation commerciale et industrielle, soumis à la concurrence - limitée - de la vente de rue; entre le $\mathrm{XVI}^{\mathrm{e}}$ et le $\mathrm{XVII}^{\mathrm{e}}$ siècle, à Florence et à Londres, les apothicaires développent des techniques et des stratégies de distinction pour susciter la confiance du chaland. Parmi elles, le dispositif qui sépare partiellement la revente, le stockage et surtout la production, entre boutique achalandée et arrière-boutique, vise à susciter l'adhésion du consommateur ${ }^{12}$. Les chirurgiens ne sont pas en reste : ils choisissent, pour leurs instruments, des matériaux sélectionnés pour leurs propriétés de dureté, de flexibilité, d'ajustage, pour les aciers, mais aussi pour leur prestige, comme l'écaille ou les pierres précieuses ornementales, adaptant ainsi des dispositifs techniques à l'usage thérapeutique qu'ils en ont et à la qualité sociale de la clientèle ${ }^{13}$. Les fabricants et les praticiens offrent ainsi des consultations avant et après la vente de leurs services, sans hésiter à adapter et combiner leurs techniques de soins dans une plus large gamme et à les adapter aux capacités financières et à la demande sociale ${ }^{14}$. En bref, la perspective commerciale a ainsi conduit à requalifier les pratiques médicales et à les intégrer dans une société de consommation en émergence, hors de la simple interlocution patient-praticien. Qui plus est, elle a conduit à enquêter sur la transformation des techniques médicales à l'époque moderne. 


\section{La fabrique des savoir-faire médicaux, entre science et technique}

L'exercice médical repose sur des théories, mais aussi sur tout un ensemble de pratiques thérapeutiques faites de matériaux et de gestes. Leur transformation au cours de l'époque moderne a fait l'objet de nombreux travaux en histoire des sciences, en histoire économique et sociale qui, adoptant un " tournant pratique ", ont conduit à une profonde révision de l'organisation productive, de la place des acteurs - praticiens, usagers dans l'activité médicale, des transformations de conceptions médicales, à partir d'une analyse de l'innovation médicale.

L'innovation thérapeutique de l'Europe moderne a partie liée avec la conquête des Nouveaux Mondes. La médecine, au cours de la période, s'est enrichie de matériaux issus de la collecte et de la transformation de plantes. Au cœur de ces transformations, la découverte des espaces lointains, en priorité les Amériques, puis leur conquête et leur prédation économique ont bousculé les savoir-faire médicinaux : telle est la thèse, cardinale, de Samir Boumediene, qui renouvelle en profondeur l'histoire médicale et politique de l'Europe moderne $^{15}$. Selon ses travaux, la couronne espagnole conduit, à l'occasion des réformes politiques des années 15701580, de vastes enquêtes de connaissance $\mathrm{du}$ territoire à des fins de domination, mais aussi d'exploitation des richesses naturelles, au nombre desquelles il faut compter les plantes médicinales et, partant, le savoir des Indiens qui les connaissent et en font usage. La colonisation espagnole transforme ainsi dura- blement la thérapeutique européenne par la quête de plantes nouvelles et, plus encore, leur intégration dans la materia medica européenne. Sur le modèle espagnol, les plantes médicinales sont un des objets de la conquête commerciale et mili-

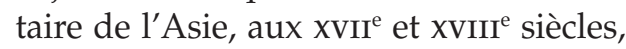
$\mathrm{du}$ fait de l'activité soignante et commerciale des chirurgiens navigants, notamment parmi les employés de la Compagnie des Indes orientales hollandaise $(\mathrm{VOC})^{16}$. C'est ainsi que les remèdes exotiques, au nombre desquels le bois de gaïac (holywood) pour soigner la syphilis et le quinquina contre les fièvres, dans leur passage du Nouveau à l'Ancien Monde en viennent à acquérir une place croissante dans la pharmacopée utilisée sur le continent et ce dès le $\mathrm{XvI}^{\mathrm{e}}$ siècle. Le succès thérapeutique et commercial des plantes médicinales du Nouveau Monde a un effet d'entraînement sur la "machine coloniale», avec le développement de procédés pour garantir leur conservation sur de longues distances, la mise en œuvre d'une certification pour « chasser les doutes et les fraudes ", à la manière des appellations d'origine contrôlée modernes, mais aussi les tentatives d'acclimatation dans les jardins d'histoire naturelle à Leyde ou au Cap, comptoir moins éloigné que les Indes orientales ${ }^{17}$. Il entraîne également la recherche de substituts continentaux, comme les remèdes chimiques.

L'essor de la médecine alchimique représente l'autre volet de l'innovation pharmaceutique de l'Europe moderne. Paracelse (1493/4?-1541), médecin formé 
dans les universités italiennes avant d'exercer dans le sud de l'Empire germanique entre Strasbourg et Nuremberg, développe un système médical trop complexe pour être exposé ici, mais un outil repose sur l'usage interne de minéraux, comme le mercure pour soigner l'hydropisie; la publication de son œuvre dans la seconde moitié $\mathrm{du} \mathrm{XVI}^{\mathrm{e}}$ siècle accélère l'exploration (al)chimique du monde minéral à des fins thérapeutiques. Ces remèdes font l'objet d'une large diffusion à l'échelle européenne, comme ce fut le cas à Londres, non sans heurts avec l'élite médicale locale ${ }^{18}$. Les cours européennes se préoccupent de découvrir de nouveaux remèdes : là, se localisent une partie de la production (al)chimique à fin thérapeutique ${ }^{19}$. Dans la Galerie des Offices, les grands-ducs François I et Ferdinand I de Medici ont établi les ateliers d'art au voisinage du laboratoire d'alchimie pour faciliter la collaboration entre artistes et hommes de science. Orfèvres, joailliers, sculpteurs, peintres et ébénistes échangent des outils, mais aussi leur savoir théorique et technique sur les matériaux et leur façon avec les alchimistes de la fonderia : les techniques picturales utilisées pour l'illustration scientifique en sont une preuve. Dans la fonderia de la Galeria dei lavori, dans un secret jalousement gardé, les Medici font élaborer aux spécialistes de " distillation » - terme qui désigne indifféremment fabrication pharmaceutique, analyse chimique et recherches alchimique - quantité de contrepoisons et de remèdes alchimiques, tandis que les ateliers contigus préparent de splendides flacons et boîtes en marqueterie de toute beauté. Leur fabrication, qui vise moins l'auto-consommation de la cour florentine que la distribution des remèdes à l'échelle européenne, comme marchandises ou comme cadeaux diplomatiques, participe à l'exercice du pouvoir du monarque, pour qui le savoir est la clef du pouvoir ${ }^{20}$.

L'innovation médicale, théorique et pratique, conduit à inclure des modes opératoires non-médicaux dans la pratique médicale que les historiens ont cherché à expliciter. Les " artisans du corps » reconfigurent savoirs médicaux et techniques par la conception d'analogies entre matériaux et opérations, entre les qualités d'une pierre précieuse et son utilisation thérapeutique ${ }^{21}$. Dans $1^{\prime}$ Italie des $\mathrm{XVI}^{\mathrm{e}}$ et $\mathrm{XVII}^{\mathrm{e}}$ siècles, les norcini (« charcutiers »), dont le nom traduit la familiarité avec les métiers de viande porcine, sont reconnus pour leurs savoirfaire concernant l'appareil urétro-génital humain : castration de jeunes garçons que l'on destine à devenir castrats, extraction des calculs de vessie, etc. ${ }^{22}$. Les écrits des chirurgiens et leur usage précis d'un vocabulaire issu de la production artisanale rendent manifestes les emprunts que ces derniers font aux métiers du cuir, de la couture, voire à l'ingénierie militaire ${ }^{23}$. L'instrumentation scientifique de l'époque bénéficie des techniques développées à des fins thérapeutiques. C'est le cas de la toute jeune science expérimentale londonienne dont la pompe à vide repose sur une maîtrise des procédés de verre soufflé pour les ventouses ${ }^{24}$; les expérimentations pharmaceutiques irriguent l'industrie alimentaire qui émerge en France à la fin du XVIII $^{\mathrm{e}}$ siècle $^{25}$. Le laboratoire, longtemps pensé comme l'espace scientifique par excellence, apparaît désormais comme le résultat du transfert du matériel et des pratiques pharmaceutiques ${ }^{26}$. Les ponts 
conceptuels et pratiques jetés entre artisanats des matériaux et médecine ont ainsi irrigué l'activité manufacturière et les sciences naturelles.

Les transformations des pratiques thérapeutiques modifient, qui plus est, théories médicales et institutions de la médecine. L'arrivée d'une materia medica nouvelle, en particulier du quinquina aux propriétés fébrifuges puissantes et des remèdes paracelsiens, fragilise la médecine humorale : ce que les contemporains appellent " remèdes spécifiques » traitent efficacement un symptôme, quelle que soit la complexion ou l'équilibre des humeurs ${ }^{27}$. Au-delà des plantes à finalité médicinale, les régions périphériques deviennent sources de nouvelles techniques thérapeutiques, à l'instar de l'inoculation. Ce procédé préventif contre la variole - dépôt de pus variolique dans une entaille cutanée fut observé dans l'Empire ottoman par une femme de diplomate anglais qui en a initié l'adoption en Angleterre et dans les colonies américaines ${ }^{28}$. Les discours médicaux s'ajustent aux pratiques auxquels ils cherchent à donner sens. Les chirurgiens qui usent d'instruments localisent les pathologies qu'ils soignent dans le corps malade et adoptent une perspective mécaniste dans la compréhension de la physiologie humaine, ce qui révolutionne la pensée médicale ${ }^{29}$. Ầ la fin $\mathrm{du} \mathrm{xvIII}^{\mathrm{e}}$ siècle, les praticiens convoquent des théories médicales distinctes, sinon concurrentes pour justifier l'usage de l'appareillage médico-électrique, en fonction des analogies faites, en médecine interne, aux remèdes et aux bains, ou à l'application d'instruments de chirurgie, les parties du corps humain devenant ainsi partie intégrante du dis- positif technique ${ }^{30}$. Les régimes de santé, genre médical étudié au regard de la culture matérielle de la Renaissance italienne, formulent des conseils en matière d'hygiène de vie et d'adaptation des objets du quotidien, afin de contrôler la qualité de l'eau, de l'air et leurs effets sur la santé : ils traduisent sur le papier l'ajustement matériel et savant des discours médicaux et des comportements habituels des classes aisés ${ }^{31}$. À la fin de la période, c'est l'environnement industriel lui-même qui fait l'objet des préoccupations médicales. À la suite des chirurgiens, avec les artisans, dont ils soignent les maux et les accidents, une élite philanthrope éclairée de médecins et d'administrateurs suscite une prise de conscience sanitaire, relayée par les institutions savantes - Société royale de médecine, Académie des sciences - et fait émerger une réflexion sur la santé des travailleurs de l'industrie, avant que la nouvelle politique industrielle de l'Empire ne la fasse disparaître ${ }^{32}$. Les techniques thérapeutiques ont ainsi contribué au renouvellement théorique médical moderne.

À la lecture des recherches récentes, il apparait vain d'isoler, sinon artificiellement, les pratiques thérapeutiques et les savoirs médicaux d'autres techniques et savoir-faire artisanaux de l'Europe moderne. Dans celle-ci, l'innovation repose ainsi sur une longue chaîne d'acteurs - congrégations religieuses, marchands, médecins, pharmaciens, courtisans, entrepreneurs, administrateurs - dont l'activité est constitutive de la définition des techniques thérapeutiques, aux implications politiques qu'il nous faut maintenant considérer. 


\section{Les techniques de la médicalisation}

La médicalisation des sociétés européennes dispose de plusieurs acceptions chez les historiens modernistes : l'une est descriptive et désigne la densité de soignants et d'équipements sanitaires en fonction de l'espace ou de la démographie; l'autre désigne un processus par lequel le comportement des hommes devient soumis à une autorité médicale aisément conçue comme coercitive ${ }^{33}$. C'est cette dernière acception que l'histoire des techniques thérapeutiques contribue à requalifier.

Équipement urbain de premier ordre, l'hôpital a retrouvé ses fonctions soignantes et au cœur des dispositifs de soin à l'époque moderne, selon des gradients qui varient entre les grandes capitales européennes - Florence, Paris, Londres - et les petites villes. Michel Foucault, en effet, avait argué que l'hôpital, avant la fin du xvIII ${ }^{\mathrm{e}}$ siècle, n'est pas une "machine à guérir », au sens où sa fonction n'est pas de soigner, d'exercer une action thérapeutique, mais d'exclure et de contenir une population pauvre. Cette chronologie, tenace, est désormais abandonnée grâce à une série de travaux cohérents sur l'ensemble de l'espace européen : les archives administratives et comptables sur les hôpitaux du sud et du nord de l'Europe ont conduit à sa complète révision. Dès le $\mathrm{xvI}^{\mathrm{e}}$ siècle, les efforts des administrateurs et des soignants visent à proposer de nouveaux traitements à la patientèle : achat de nouveaux produits, comme le bois de gaïac que les Fugger fournissent en grande quantité aux hôpitaux de Nuremberg; arsenal thérapeutique complet à l'hôpital Santa Maria Nuova de Florence, qui s'adjoint aux missions pastorales de l'hôpital et qui sert de modèle aux grands hôpitaux londoniens; les essais de nouvelles thérapies à l'Hôtel-Dieu de Marseille ${ }^{34}$. Les nombreuses activités qu'il abrite font également de l'hôpital un lieu privilégié de l'histoire des techniques entendue tant du côté de l'histoire industrielle - process alimentaire; évaluation de la qualité des produits qu'il produit ou échange à des fins thérapeutiques, etc. - que de la comptabilité $^{35}$. L'hôpital, dans les grandes villes européennes, devient non seulement un centre thérapeutique, mais le lieu d'importantes innovations en matière de techniques administratives.

L'espace domestique a lui aussi retrouvé une fonction majeure de l'élaboration médicinale moderne. La domesticité curiale y participe : à la périphérie de la zone d'influence des Medici, dans l'Empire germanique de la fin $d u x v I^{e}$ siècle, les femmes nobles contribuent activement à la recherche et à la production (al)chimiques ${ }^{36}$. Pour les populations plus modestes, l'espace domestique reste le site privilégié de la production de remèdes à fin d'autoconsommation mais aussi de revente ${ }^{37}$. Hors des intérieurs aisés de l'Italie renaissante, dont on a vu comment les régimes de santé participaient à leur configuration, les techniques médicales transforment l'aménagement des maisons bourgeoises : alors que les premiers appareils pour bains électriques, à la fin du XvIII ${ }^{e}$ siècle, indiqués pour atténuer les paralysies, occupent plusieurs pièces du logement, patients, médecins et fabricants collaborent pour limiter les désagré- 
ments du fonctionnement de la machine et de ses dysfonctionnements, mais aussi pour miniaturiser l'appareil et le rendre propre à l'usage personnel des patients. La maison est un lieu de conception et d'ajustement où les patients tiennent un rôle majeur en participant pleinement à l'élaboration technique.

La médicalisation repose sur des techniques de papier (paper technologies) qui visent à collecter, codifier et diffuser l'information médicale : il s'agit ainsi des tableaux pré-imprimés pour consigner les observations, des dossiers de patients, les index, des revues et bibliothèques spécialisées ${ }^{38}$. Il faut souligner ici la place particulière des techniques visuelles, les estampes notamment, dans les dispositifs qui façonnent l'interprétation médicale des savoir-faire ${ }^{39}$. Faisant feu de tout bois - cerisier, cire, mais aussi morceaux de corps préparés - les modèles anatomiques sont indissociables de la conception mécaniste des corps qui se développe au $\mathrm{XVIII}^{\mathrm{e}}$ siècle. Leur succès tient à la variété des consommations dont ils font l'objet, privées, publiques, muséales, savantes, professionnelles et érotiques. Une large population y a accès, depuis la cour viennoise jusqu'aux sages-femmes rurales formées par $\mathrm{M}^{\text {me }} \mathrm{du}$ Coudray, en passant par les citadins aisés qui se rendent dans des musées de Florence, Vienne ou Londres ${ }^{40}$. La publicité dont ils font l'objet repose sur de subtils jeux de communication : Fredryk Ruysch, célèbre préparateur anatomique à Amsterdam, ne rend pas compte de ses secrets de fabrication dans les publications vantant la qualité et la beauté de ses produits, ce qui lui garantit un quasi quasi-monopole sur le marché ${ }^{41}$. La visualisation des corps, tout autant que les techniques de papier, ont contribué au façonnage du "regard" médical (M. Foucault), des praticiens, mais aussi des patients.

Les patients " comptent " dans l'élaboration des techniques médicales, mais comment ${ }^{42}$ ? Si $l^{\prime}$ historiographie s'accorde sur la part de l'usager de médecine, une ligne de fracture interprétative existe entre ce qui ressort de l'assujettissement et de la pleine capacité à agir (agency). Pour nombre d'historiens, le patient bien nommé équivaut à un simple matériau d'expérience. La « colonisation des savoirs médicinaux » repose sur les corps, supports de la lente élaboration du remède : corps des Indiens soumis à la question des tribunaux inquisitoriaux à Lima, Mexico puis Cartagena de Indias, qui s'intéressent à l'usage des plantes dans la mesure où il est indissociable de l'idolâtrie; corps des enquêteurs qui goûtent, expérimentent sur eux-mêmes; corps des Indianos - Espagnols acclimatés aux Indes - qui cherchent activement des remèdes; corps des pauvres patients de l'hôpital Santo Spirito de Rome; corps des monarques anglais et français, dont les goûts déterminent ceux de la cour. Ces femmes et ces hommes dotés d'entendement et de parole sont le support de la lente élaboration du remède. Le médicament, à la fois matériau et savoir, représente le résultat d'opérations et d'expérience pour définir les indications, les posologies ou dosages ainsi que les formes galéniques (poudres, sirops, clystères $)^{43}$. Lors de ces expériences, la totalité du corps social est mis à l'épreuve, même si le risque était distribué de façon inégale entre les populations pauvres et aisées, en utilisant les patients hospitaliers ou les prisonniers comme « corps 
vils » sur lesquels faire peser le danger ${ }^{44}$. La médicalisation des matériaux irait ainsi de pair avec une déshumanisation partielle du soin. L'étude de l'inoculation prolonge cette discussion : valorisation de l'autogouvernement rationnel de soi - lorsque les élites choisissent de s'administrer le virus à elles-mêmes -, la technique devient un mode d'administration des populations pesant singulièrement sur les populations sujettes, esclaves des colonies en prioritét5. Ses partisans qui justifient sa mise en œuvre par une rationalité probabiliste, transforment ainsi la conception morale de la vie et contribuent à l'émergence du risque comme mode de gouvernement des conduites.

$A$ contrario d'autres études établissent comment se construit l'intelligence technique de compréhension et d'adaptation des techniques. Les patients des Blakey rapportent ainsi dans leur correspondance comment ils développent des gestes pour ajuster leurs bandages herniaires et les réparer; ils suggèrent des modifications de l'équipement; ils participent à l'établissement des mesures de leur corps et de la flexibilité requise de leurs bandages, en inventant des dispositifs de notation ou de codage pour l'adaptation du produit ${ }^{46}$. En ce sens, les patients sont à la fois usagers, agents et prescripteurs de techniques. Mieux, ils contribuent à transformer les institutions charitables sur le modèle hospitalier : tel est la thèse de Kevin Siena qui étudie les patients syphilitiques à Londres. Après l'incendie de 1666 qui prive les hôpitaux royaux Guy's et St Thomas de revenus, les administrateurs requièrent le paiement de la population londonienne pour l'accès aux services hospitaliers, désormais réservés en pratique aux hommes relativement aisés. De fait, les patients qui en ont les moyens s'achètent la discrétion et les services de médecins particuliers, dont on trouve les réclames dans la presse. Mais, dans une métropole en pleine expansion où le service domestique permet la constitution d'un petit capital pour les jeunes femmes originaires des campagnes, la maladie affecte non seulement le corps, mais elle menace aussi le maintien au travail et les perspectives d'avenir. Les demandes de soin qu'elles formulent de manière insistante auprès des paroisses, conduisent à la reconfiguration des workhouses, construites dans les années 1720 pour enfermer les pauvres oisifs, en cliniques dotées d'équipes soignantes, de salles dédiées aux malades et de médicaments à base de mercure ${ }^{47}$. Colin Jones, quant à lui, voit dans la marchandisation, ouverte et relativement égalitaire qui se tisse dans les Affiches provinciales, une remise en cause radicale des hiérarchies du corps social; la " grande chaîne de l'achat ", entre Révolution commerciale et Révolution politique, conduit à l'émancipation politique des patients ${ }^{48}$. En ce sens, la médicalisation, dans son acception de qualification médicale des matériaux et des gestes, est un des lieux où se négocient les pouvoirs de l'Europe moderne.

Le " carrefour thérapeutique ", à l'intersection d'approches distinctes en histoire économique et sociale et en histoire des sciences, a ainsi ouvert des voies pour comprendre les rapports entre techniques, sociétés et pouvoirs en Europe et à ses marges au cours de l'époque moderne. Par son biais, les historiens ont entrepris d'analyser les processus matériels, savants et sociaux par lesquels 
des biens et des pratiques ont acquis une signification médicale, processus lents, heurtés, contestés, par lesquels fabricants, métiers médicaux, consommateurs et gouvernements ont tous joué des rôles déterminants. La médecine représente ainsi un espace d'échange matériel, savant, social et politique qui participe à la construction des sociétés européennes.

Ces travaux, lus par le prisme de l'histoire des techniques, invitent, en retour, à comprendre en quoi ce " carrefour thérapeutique » contribue à l'histoire des techniques de l'Europe moderne. L'incorporation de matériaux dans la pharmacopée moderne - nouvelles plantes américaines, minéraux, fluide électrique - fut à la fois le résultat d'initiatives individuelles d'entrepreneurs proposant par la commercialisation un usage médical, mais aussi de politiques visant à capter des savoirs et des savoirfaire à des fins de gouvernement. Cette vaste entreprise de médicalisation du monde a reposé sur une profonde transformation des techniques d'information, ces "techniques de papier " qui organisent et transmettent les savoirs au sein des métiers médicaux, mais aussi parmi une population élargie d'administrateurs, de notables et de classes intermédiaires, cette " heureuse médiocrité " (Colin Jones) de consommateurs de médecine. L'usage, mais aussi l'élaboration des techniques médicales reposent ainsi sur un large éventail d'acteurs, des patients eux-mêmes dont les corps sont le support d'expériences volontaires ou subies, qui s'approprient et adaptent les objets et les savoir-faire, aux agents de gouvernement, en passant par de nombreux artisans aux métiers divers et les praticiens de médecine. Leur rôle d'iden- tification des besoins, d'information, d'ajustement et de promotion est désormais réévalué. En un sens, la médicalisation, entendue non comme imposition d'un pouvoir, mais comme croyance en un système technique de rétablissement de la santé, transforment les gouvernements modernes.

\section{Notes}

1. John V. Pickstone (éd.), Medical innovations in historical perspective, Houndmills, Macmillan, 1992.

2. Ruth Schwartz Cowan, "The consumption junction : a proposal for research strategies in the sociology of technology ", in E. WIEBE et al. (éd.), The social construction of technological systems : new directions in the sociology and history of technology, Cambridge, Mass., Harvard University Press, 1987, p. 261-280.

3. Roy PORTER, «The patient's view : doing medical history from below », Theory and society, $\mathrm{n}^{\circ} 14$ (1985), p. 175-198; id.. Health for sale : quackery in England, 1660-1850, Manchester, Manchester University Press ND, 1989.

4. David Gentilcone, Medical charlatanism in early modern Italy, Oxford, Oxford University Press, 2006.

5. Pour une bibliographie plus complète jusqu'à 2013, je renvoie à mon introduction « The crafting of medicine in the early industrial age ", Technology $\mathcal{E}$ Culture, $\mathrm{n}^{\circ} 54$ (2013), p. 429-437.

6. James Harvey Young, «Patent medicines : an early example of competitive marketing ", The Journal of economic history, $\mathrm{n}^{\circ}$ 20-4 (1960), p. 648-456; Alan MACKINTOSH, « The patent medicines industry in late Georgian England : a respectable alternative to both regular medicine and irregular practice ", Social history of medicine, 28 mai 2016,<doi : 10.1093/ shm/hkw054>.

7. Mary FISSELL, «The marketplace of print », in Mark S. R. Jenner, Patrick WALlis (éd.), Medicine and the market in England and its colonies, c. 1450-c. 1850, Basingstoke, Palgrave Macmillan, 2007, p. 108-132.

8. Robert Weston, Medical consulting by letter in France, 1665-1789, Farnham, Ashgate, 2013.

9. Liliane Hilaire-Pérez, Christelle Rabier, "Self-machinery? Steel trusses and the management of ruptures in eighteenth-century Europe ", Technology E Culture, ${ }^{\circ} 54$ (2013), p. 460-502.

10. Laurence W. BRockliss, Colin Jones, The medical world of early modern France, Oxford, Clarendon Press, 1997, not. p. 652. 
11. Samir Boumediene, La colonisation du savoir, Vaulx-en-Velin, Les éditeurs des mondes à faire, 2016.

12. Patrick WALlis, "Consumption, retailing and medicine in early modern London ", Economic history review, $\mathrm{n}^{\circ} 61$ (2008), p. 26-53; James SHAw, Evelyn Welch, Making and marketing medicines in Renaissance Florence, Amsterdam, Rodopi, 2011; Christelle RABIER, « Les circulations techniques médicales entre Europe et colonies, 1600-1800 : l'apport de la perspective commerciale ", in Pilar González Bernaldo de Quirós, Liliane HilairePérez (éd.), Les savoirs-mondes : mobilités et circulation des savoirs depuis le Moyen Âge, Rennes, PUR, 2015, p. 227-234.

13. John KIRKUP, The evolution of surgical instruments : an illustrated history from ancient times to the twentieth century, Novato, CA, Norman Publishing, 2006.

14. Lynn Sorge-English, Stays and body image in London : the staymaking trade, 1680-1810, London, Pickering \& Chatto, 2011; Alun Withey,Technology, self-fashioning and politeness in eighteenth-century Britain refined bodies, London, Palgrave Macmillan, 2015.

15. S. Boumediene, La colonisation du savoir, op. cit.

16. Harold J. CooK, Matters of exchange. Commerce, medicine and science in the Dutch Golden Age, New Haven, Conn., Yale University Press, 2007; Chakrabarti Pватік, Materials and medicine : trade, conquest and therapeutics in the eighteenth century, Manchester, Manchester University Press, 2010.

17. James E. McClellan, François Regourd, The colonial machine : French science and overseas expansion in the Old Regime, Turnhout, Brepols, 2011; Matthew James CRAWFORD, " "Para desterrar las dudas y adulteraciones" : scientific expertise and the attempts to make a better bark for the royal monopoly of quina (1751-1790) », Journal of spanish cultural studies, $\mathrm{n}^{\circ} 8$ (2007), p. 193-212; H. J. CooK, Matters of exchange..., op. cit., p. 308 et 325.

18. Deborah E. HARKNEss, The jewel house: Elizabethan London and the scientific revolution, New Haven London, Yale University Press, 2007.

19. Pamela H. Sмiтh, The body of the artisan. Art and experience in the scientific revolution, Chicago, The University of Chicago Press, 2004; Ursula KLEIN, " Apothecaries' shops, laboratories and chemical manufacture in eighteenth-century Germany ", in Lissa Roberts, Simon Schaffer, Peter Dear (éd.), The mindful hand. Inquiry and invention from the late Renaissance to early industrialisation, Amsterdam, Knaw, 2007, p. 248-276.

20. Fanny KIEFFER, " The laboratories of art and alchemy at the Uffizi Gallery in Renaissance Florence : some material aspects ", in Sven Dupré (éd.), Laboratories of art : alchemy and art technology from antiquity to the eighteenth century, Cham, Springer, 2014, p. 105-127.

21. Sandra Cavallo, Artisans of the body in early modern Italy : identities, families and masculinities, Manchester, Manchester University Press, 2007.

22. D. Gentilcore, Medical charlatanism in early modern Italy, op. cit.

23. S. CAVAllo, Artisans of the body in early modern Italy..., op. cit., ; Donatella BARTOLINI, « On the borders : surgeons and their activities in the Venetian State (1540-1640) ", Medical history, n 59 (2015), p. $83-100$.

24. Terje BRUNDTLAND, "From medicine to natural philosophy : Francis Hauksbeea's way to the air-pump ", British Journal for the history of science, $n^{\circ}$ 41-2 (2008), p. 209-240.

25. Emma C. Spary, Feeding France : new sciences of food, 1760-1815, Cambridge, Cambridge University Press, 2014.

26. Ursula KLEIN, « The laboratory challenge : some revisions of the standard view of early modern experimentation ", Isis, n 99 (2008), p. 769-782.

27. Harold J. CooK, " Markets and culture : medical specifics and the reconfiguration of the body in early modern Europe ", Transactions of the Royal historical Society, $\mathrm{n}^{\circ} 21$ (2011), p. 123-145.

28. Pour une analyse sur les modalités d'adoption sur le continent européen, Jean-Baptiste Fressoz, L'apocalypse joyeuse : une histoire du risque technologique, Paris, Éditions du Seuil, 2012, p. 27-68.

29. Oswei TEMKIN, "The role of surgery in the rise of modern medical thought ", Bulletin for the history of medicine, $\mathrm{n}^{\circ} 25$ (1951), p. 248-259.

30. François ZANETTI, «Curing with machines : medical electricity in eighteenth-century Paris ", Technology \& Culture, $\mathrm{n}^{\circ} 54$ (2013), p. 503-530.

31. Sandra Cavallo, Tessa Storey, Healthy living in late Renaissance Italy, Oxford, Oxford University Press, 2013; Sandra CAvallo, " Health, air and material culture in the early modern italian domestic environment ", Social history of medicine, mai 2016 [en ligne], <hkw029. doi : 10.1093/shm/hkw029>.

32. Christelle RABIER, "Le "service public" de la chirurgie : administration des premiers secours et pratiques professionnelles à Paris au $\mathrm{XVIII}^{\mathrm{e}}$ siècle ", Revue d'histoire moderne et contemporaine, vol. 58, $n^{\circ} 1$ (2011), p. 101-127; Thomas LE Roux, « L'effacement du corps de l'ouvrier. La santé au travail lors de la première industrialisation de Paris (1770-1840) ", Le Mouvement social, vol. 234, $\mathrm{n}^{\circ} 1$, 2011, p. 103-119.

33. Dans le premier sens, Jean-Pierre GoubERT, "Réseau médical et médicalisation en France à la fin du XVIII ${ }^{\mathrm{e}}$ siècle ». Annales de Bretagne et des pays de l'Ouest, vol. 86, $\mathrm{n}^{\circ}$ 2, p. 221-29; dans le second, 
Michel Foucault, « Histoire de la médicalisation », Hermès, vol. 2, n² 2, p. 11-29.

34. Claudia SteIN, Negotiating the French pox in early modern Germany, Farnham, Ashgate, 2009; John Henderson, The Renaissance hospital : healing the body and healing the soul, New Haven, Yale University Press, 2006; Judith AzIzA, Soigner et être soigné sous l'Ancien Régime. L'Hôtel-Dieu de Marseille aux XVII et XVIII siècles, Aix-en-Provence, Presses universitaires de Provence, 2013.

35. E. C. SPARY, Feeding France: new sciences of food, 1760-1815, op. cit.; Pierre-Charles Pradier, " Les bénéfices terrestres de la charité. Les rentes viagères des hôpitaux parisiens, 1600-1690 ", Histoire et mesure, XXVI (2011), p. 31-76.

36. Alisha RANKIN, Panaceia's daughters : noblewomen as healers in early modern Germany, Chicago, University of Chicago Press, 2013.

37. Elaine Leong, "Making medicines in the early modern household ", Bulletin of the history of medicine, $\mathrm{n}^{\circ} 82$ (2008), p. 145-168.

38. Andrew J. Mendelsohn, Volker Hess, « Case and series : medical knowledge and paper technology, 1600-1900 », History of science, $\mathrm{n}^{\circ} 48$ (2010), p. 287-314; Emmanuelle Chapron, " Ad utilità pubblica ". Politique des bibliothèques et pratiques du livre à Florence au XVIII siècle, Genève, Droz, 2009. Michel Foucault (Naissance de la clinique. Une archéologie du regard médical, Paris, Vrin, 1961) ne s'intéresse pas à la matérialité des pratiques d'information de ce regard.
39. Sachiko Kusukawa, Picturing the book of nature: image, text and argument in sixteenth-century human anatomy and medical botany, Chicago, Chicago University Press, 2012.

40. Anna MAERKER, Model experts : wax anatomies and enlightenment in Florence and Vienna, 1775-1815, Manchester, Manchester University Press, 2011.

41. Dániel MARGócsY, "Advertising cadavers in the Republic of Letters : anatomical publications in the early modern Netherlands ", The british journal for the history of science, $42, \mathrm{n}^{\circ} 2$ (2009), p. 187-210.

42. En sociologie des techniques, Nelly Oudshoorn, Trevor Pinch (éd.), How users matter : the co-construction of users and technology, Cambridge, Mass., MIT Press, 2000.

43. F. Zanetti, " "Curing with machines... ", op. cit.

44. S. Boumediene, La colonisation $d u$ savoir, op. cit.; Grégoire Chamayou, Les corps vils. Expérimenter sur les êtres humains aux XVIII et XIXe siècles, Paris, La Découverte, 2008; F. ZANETTI, «Curing with machines... », op. cit.

45. J.-B. Fressoz, L'apocalypse joyeuse..., op. cit.

46. L. Hilaire-Pérez, C. Rabier, " Selfmachinery?... », op. cit.

47. Kevin P. SIENA, Venereal disease, hospitals and the urban poor: London's "foul wards", 1600-1800, Rochester, NY, University of Rochester Press, 2004.

48. C. JONES, op. cit. 